\title{
Synovial Tissue Sublining CD68 Expression Is a Biomarker of Therapeutic Response in Rheumatoid Arthritis Clinical Trials: Consistency Across Centers
}

\author{
BARRY BRESNIHAN, ELIZA PONTIFEX, ROGIER M. THURLINGS, MARJOLEIN VINKENOOG, \\ HANI EL-GABALAWY, URSULA FEARON, OLIVER FITZGERALD, DANIELLE M. GERLAG, TERENCE ROONEY, \\ MARLEEN G. van de SANDE, DOUGLAS VEALE, KOEN VOS, and PAUL-PETER TAK
}

\begin{abstract}
Objective. To determine whether the correlation between the mean change in disease activity and the mean change in synovial sublining (sl) CD68 expression could be demonstrated across different academic centers.

Methods. Synovial biopsies obtained at arthroscopy from patients with rheumatoid arthritis before and 160 days after rituximab therapy were selected and coded. Paired sections were processed independently at Amsterdam Medical Center (AMC) and at St. Vincent's University Hospital (SVUH), Dublin. Digital image analysis (DIA) was employed at both centers to quantify sublining CD68 expression.

Results. After analysis of CD68sl expression at centers in 2 different countries, high levels of intracenter and intercenter agreement were observed. For the pooled sections stained at AMC, the correlation between 2 investigators was $\mathrm{R}=0.942, \mathrm{p}=0.000$, and for sections stained at $\mathrm{SVUH}, \mathrm{R}=$ $0.899, \mathrm{p}=0.001$. Similarly, the intracenter correlations for $\Delta \mathrm{CD} 68 \mathrm{sl}$ expression after treatment were $\mathrm{R}=0.998, \mathrm{p}=0.000$, for sections stained at $\mathrm{AMC}$ and $\mathrm{R}=0.880, \mathrm{p}=0.000$, for sections stained at $\mathrm{SVUH}$. The intercenter correlation for the pooled scores of sections stained at AMC was $\mathrm{R}=0.85$, $\mathrm{p}=0.000$, and for the sections stained at SVUH, $\mathrm{R}=0.62, \mathrm{p}=0.001$. The consistent correlation between $\triangle \mathrm{DAS}$ (Disease Activity Score) and $\triangle \mathrm{CD} 68 \mathrm{sl}$ expression across different studies (Pearson correlation $=0.895, \mathrm{p}<0.001)$ was confirmed. The standardized response mean values for $\Delta \mathrm{CD} 68 \mathrm{sl}$, calculated from analyses at both AMC and SVUH, were consistently 0.5 or greater, indicating a moderate to high potential to detect change.

Conclusion. The correlation between mean $\triangle \mathrm{DAS}$ and mean $\triangle \mathrm{CD} 68 \mathrm{~s}$ l expression was confirmed across 2 centers. Examination of serial biopsy samples can be used reliably to screen for interesting biological effects at the site of inflammation at an early stage of drug development. (J Rheumatol 2009;36:1800-2; doi:10.3899/jrheum.090348)
\end{abstract}

Key Indexing Terms:

SYNOVIUM MACROPHAGES RHEUMATOID ARTHRITIS BIOMARKERS

An OMERACT special interest group consisting of researchers with expertise in synovial tissue analysis was established in 2004. A considerable evidence base has accu- mulated at one academic center that supports the hypothesis that synovial tissue sublining (sl) CD68 expression may be a biomarker of clinical response to therapeutic intervention
From the Department of Rheumatology, St. Vincent's University Hospital, and The Conway Institute of Biomolecular and Biomedical Research, University College Dublin, Dublin, Ireland; Division of Clinical Immunology and Rheumatology, Academic Medical Center/University of Amsterdam, Amsterdam, The Netherlands; University of Manitoba, Winnipeg, Canada; and University of California at San Francisco, San Francisco, USA.

B. Bresnihan, MD, FRCP, Professor of Rheumatology, Department of Rheumatology, St. Vincent's University Hospital and The Conway Institute of Biomolecular and Biomedical Research, University College Dublin; E. Pontifex, MB, FRACP, Research Fellow, Department of Rheumatology, St. Vincent's University Hospital; R.M. Thurlings, MD, Research Fellow; M. Vinkenoog, PhD, Project Scientist, Division of Clinical Immunology and Rheumatology, Academic Medical Center/University of Amsterdam; H. El-Gabalawy, MD, PhD, Professor of Rheumatology, University of Manitoba; U. Fearon, PhD, Senior Scientist, Department of Rheumatology, St. Vincent's University Hospital and The Conway Institute of Biomolecular and Biomedical Research, University College Dublin;
T. Rooney, MB, MRCPI, Research Fellow, University of California at San Francisco; O. FitzGerald, MD, FRCPI, Newman Professor of Rheumatology, Department of Rheumatology, St. Vincent's University Hospital and The Conway Institute of Biomolecular and Biomedical Research, University College Dublin; D. Gerlag, MD, Assistant Professor of Medicine; M.G. van de Sande, MD, Research Fellow, Division of Clinical Immunology and Rheumatology, Academic Medical Center/University of Amsterdam; D. Veale, MD, FRCP, Professor of Rheumatology, Department of Rheumatology, St. Vincent's University Hospital and The Conway Institute of Biomolecular and Biomedical Research, University College Dublin; K. Vos, MD, PhD, Rheumatologist, Division of Clinical Immunology and Rheumatology, Academic Medical Center/University of Amsterdam; P-P. Tak, MD, PhD, Professor of Medicine, Director, Division of Clinical Immunology and Rheumatology, Academic Medical Center/University of Amsterdam.

Address reprint requests to Dr. B. Bresnihan, St. Vincent's University Hospital, Dublin 4, Ireland.E-mail: barry.bresnihan@gmail.com 
in patients with rheumatoid arthritis (RA), an observation of potential utility in early phase randomized clinical trials $(\mathrm{RCT})^{1,2}$. The available data were presented at the OMERACT meeting in $2006^{3}$ and were comprehensively reviewed more recently ${ }^{4}$. Employing a range of effective therapeutic agents, including prednisolone, methotrexate, leflunomide, and infliximab, a consistent correlation between the mean change in disease activity and the mean change in synovial sublining CD68 ( $\triangle \mathrm{CD} 68 \mathrm{sl})$ expression was observed. Moreover, patient cohorts that received either placebo, stable doses of a disease modifying antirheumatic drug (DMARD), or an ineffective compound demonstrated no change in CD68sl expression. Thus, in relatively small patient cohorts, effective therapies were readily distinguished from ineffective compounds by an analysis of synovial tissue, the primary target of disturbed immunomodulatory pathways in RA. More recent published studies have supported these earlier findings ${ }^{5-7}$.

Following OMERACT 2006, the primary research objective proposed for presentation at OMERACT 2008 was to determine whether the correlation between the mean change in disease activity and the mean $\triangle \mathrm{CD} 68 \mathrm{sl}$ expression could be demonstrated across different academic centers.

\section{MATERIALS AND METHODS}

For the purposes of our study, synovial biopsies obtained at arthroscopy from the same joints before and 160 days after 2 standard infusions of rituximab at the Academic Medical Center (AMC), Amsterdam, were select$\mathrm{ed}^{8,9}$. All patients had active seropositive RA ${ }^{11}$. The Disease Activity Score 28 (DAS28) $^{11}$ was determined at each of the 2 timepoints. Paired sections selected from each biopsy were coded and processed independently at AMC and at St. Vincent's University Hospital (SVUH), Dublin, as described $^{8,9,12}$. There were some methodological differences in tissue preparation and staining techniques. The reagent kits and antibodies were sourced from the same company (Dako) at both centers. Optimum primary antibody dilutions were determined locally. Incubation times were similar in both centers.

Digital image analysis (DIA) was employed at both centers to quantify CD68sl expression. The methodology has been described ${ }^{12,13}$. At AMC, CD68sl scores were derived from an analysis of 18 images acquired from each biopsy section and were expressed as the mean number of positively stained cells per square millimeter ${ }^{13}$. At SVUH, the scores were derived from an analysis of 6 images from each biopsy section and expressed as the mean area of tissue occupied by positively stained cells ${ }^{12}$. When quantifying CD68sl expression, the investigators at each center were unaware of patients' identities, therapeutic responses, or time sequence of each stained section.

In order to evaluate intracenter variation, 2 investigators (RT and MV) from AMC independently quantified CD68sl expression, and the results were compared. In order to evaluate intercenter variation, an investigator from SVUH (EP) independently quantified CD68sl expression in all sections, and results were compared to those derived by RT. Thus, after decoding, intercenter and intracenter variations in quantification of CD68sl expression, $\Delta \mathrm{CD} 68 \mathrm{sl}$, and the relationships between $\Delta \mathrm{CD} 68 \mathrm{sl}$ and $\triangle \mathrm{DAS}$ were determined.

Determination of the sensitivity of biomarker expression to detect change was based on the standardized response mean (SRM), which is calculated by dividing the mean change by the standard deviation of the mean change. SRM $>0.8$ indicates a high potential to detect change, and SRM of 0.5 indicates moderate potential.

\section{RESULTS}

The intracenter correlations for quantification of CD68sl expression were excellent. For the pooled sections stained at $\mathrm{AMC}$, the correlation between 2 investigators was $\mathrm{R}=$ $0.942, \mathrm{p}=0.000$, and for sections stained at SVUH, $\mathrm{R}=$ $0.899, \mathrm{p}=0.001$. Similarly, the intracenter correlations for $\Delta \mathrm{CD} 68$ sl expression after treatment were $\mathrm{R}=0.998$ with a $\mathrm{P}=0.000$ for sections stained at $\mathrm{AMC}$ and $\mathrm{R}=0.880$ with a $\mathrm{P}=0.000$ for sections stained at SVUH.

The intercenter correlation for the pooled scores of sections stained at $\mathrm{AMC}$ was $\mathrm{R}=0.85, \mathrm{p}=0.000$, and for the sections stained at $\mathrm{SVUH}, \mathrm{R}=0.62, \mathrm{p}=0.001$. The intercenter correlation for $\triangle \mathrm{CD} 68 \mathrm{sl}$ scores derived from sections stained at $\mathrm{AMC}$ was $\mathrm{R}=0.67, \mathrm{p}=0.013$, but only $\mathrm{R}=0.22$ (not statistically significant) for the $\triangle \mathrm{CD} 68 \mathrm{sl}$ scores derived from sections stained at SVUH.

This discrepancy could be explained by fading of the staining intensity in some sections during an unavoidable delay in acquiring images and quantifying CD68sl expression in sections transported from Dublin to Amsterdam due to an unforeseen illness of one of the investigators. Thus, some of the sections that were stained and images recorded at SVUH demonstrated prominent CD68sl expression, but minimal expression when the images were acquired at AMC some weeks after staining. This phenomenon resulted in inconsistent determination of $\Delta \mathrm{CD} 68 \mathrm{sl}$ values for some sections stained at SVUH and quantified at AMC. This observation further underlines the known importance of analyzing sections stained with amino ethylcarbazole within a few weeks when this dye is used for immunohistochemistry. Further analysis of this issue is in progress and will be reported separately.

The mean DAS28 at baseline was 6.7. The mean value 160 days after rituximab was 5.0 (mean $\triangle \mathrm{DAS} 28=1.7$ ). The relationships between the mean $\triangle \mathrm{CD} 68 \mathrm{sl}$ and the mean $\triangle$ DAS28 from the 4 completed analyses undertaken in this study (RT, paired AMC-stained sections, $\mathrm{n}=19$; RT, paired SVUH-stained sections, $\mathrm{n}=18$; MV, paired AMC-stained sections, $\mathrm{n}=19 ; \mathrm{MV}$, paired SVUH-stained sections, $\mathrm{n}=18$ ) were added to the previously described study cohorts ${ }^{5}$. The consistent correlation between $\triangle \mathrm{DAS}$ and $\triangle \mathrm{CD} 68$ sl expression $($ Pearson correlation $=0.895, \mathrm{p}<0.001$ ) was confirmed. In this regard, it was not feasible to compare the correlation between the $\triangle \mathrm{CD} 68 \mathrm{sl}$ values calculated at $\mathrm{SVUH}$ and $\triangle$ DAS because different units of CD68sl expression were employed.

The SRM values for $\triangle \mathrm{CD} 68 \mathrm{sl}$, calculated from the analyses at AMC, in addition to the analyses completed at SVUH (EP, paired AMC-stained sections, $\mathrm{n}=16$; EP, paired SVUH sections, $\mathrm{n}=16$ ), were consistently 0.5 or greater, indicating a moderate to high potential to detect change.

\section{DISCUSSION}

Our study was designed to determine if the correlation Personal non-commercial use only. The Journal of Rheumatology Copyright $@$ C 2009. All rights reserved. 
between the mean $\triangle \mathrm{DAS}$ and the mean $\triangle \mathrm{CD} 68 \mathrm{sl}$, consistently observed in several RCT at one academic center, could be confirmed across other centers. Paired biopsy sections obtained before and after rituximab treatment were independently processed, and CD68sl expression before and after treatment was quantified at academic centers in 2 different countries. The stained sections from each center were then exchanged, and CD68sl expression and $\Delta$ CD68sl were recalculated. After decoding, excellent intracenter and intercenter agreements were observed for the pooled CD68sl expression scores. Excellent intracenter agreement was also observed for $\Delta$ CD68sl after treatment. Measurement of CD68sl expression SRM values confirmed a moderate to high potential to detect change. Finally, a consistent correlation between the mean $\Delta \mathrm{CD} 68 \mathrm{sl}$ values, calculated by different investigators from sections that were processed in the different centers, and $\triangle \mathrm{DAS} 28$ was confirmed. In a ballot at the concluding OMERACT general assembly, 55\% of the delegates agreed with this statement while 19\% disagreed. Moreover, 59\% of the delegates agreed that CD68 expression in synovial tissue is less susceptible to a placebo effect and expectation bias than clinical evaluation, compared to $13 \%$ who disagreed.

The use of arthroscopic synovial biopsy in proof-of-concept clinical trials is feasible and safe. It allows macroscopic evaluation of the synovium. Narrow-diameter arthroscopes provide access to small joints, including the ankle, wrist, metacarpophalangeal, and proximal interphalangeal joints. The arthroscopic procedure is well tolerated, with a low complication rate. In a series of more than 2000 arthroscopic biopsy procedures at the $\mathrm{AMC}$, the complication rate was $<0.3 \%{ }^{14}$. When voting, $72 \%$ of the participants at OMERACT agreed that arthroscopic synovial biopsy was safe and well tolerated, while only $8 \%$ disagreed.

In conclusion, examination of serial biopsy samples can be used reliably to screen on the group level for interesting biological effects at the site of inflammation in an early stage of drug development. In addition to immunohistochemistry, other techniques like quantitative polymerase chain reaction $^{15}$, microarray analysis ${ }^{16}$, and proteomics may provide supportive data, although they need further validation as markers of therapeutic response. It can be anticipated that future development will include the use of more extensive markers of joint degradation as well as the use of panels of biomarkers in synovial tissue samples.

\section{REFERENCES}

1. Gerlag DM, Haringman JJ, Smeets TJ, et al. Effects of oral prednisolone on biomarkers in synovial tissue and clinical improvement in rheumatoid arthritis. Arthritis Rheum 2004;50:3783-91.
2. Haringman JJ, Gerlag DM, Zwinderman AH. Synovial tissue macrophages: Highly sensitive biomarkers for response to treatment in rheumatoid arthritis patients. Ann Rheum Dis 2005;64:834-8.

3. Bresnihan B, Gerlag DM, Rooney T, et al. Synovial macrophages as a biomarker of response to therapeutic intervention in rheumatoid arthritis: Standardization and consistency across centers. J Rheumatol 2007;34:620-2

4. Gerlag D, Tak PP. Novel approaches for the treatment of rheumatoid arthritis: Lessons from the evaluation of synovial biomarkers in clinical trials. Best Pract Res Clin Rheumatol 2008;22:311-23.

5. Wijbrandts CA, Vergunst CE, Haringman JJ, Gerlag DM, Smeets TJ, Tak PP. Absence of changes in the number of synovial sublining macrophages after ineffective treatment for rheumatoid arthritis: Implications for use of synovial sublining macrophages as a biomarker. Arthritis Rheum 2007;56:3869-71.

6. Vergunst CE, Gerlag DM, Dinant H, et al. Blocking the receptor for $\mathrm{C} 5 \mathrm{a}$ in patients with rheumatoid arthritis does not reduce synovial inflammation. Rheumatology 2007;46:1773-8.

7. Vergunst CE, Gerlag DM, Lopatinskaya L, et al. Modulation of CCR2 in rheumatoid arthritis: A double-blind, randomized, placebo-controlled clinical trial. Arthritis Rheum 2008;58:1931-9.

8. Vos K, Thurlings RM, Wijbrandts CA, van Schaardenburg D, Gerlag DM, Tak PP. Early effects of rituximab on the synovial cell infiltrate in patients with rheumatoid arthritis. Arthritis Rheum 2007;56:772-8.

9. Thurlings RM, Vos K, Wijbrandts CA, Zwinderman A, Gerlag DM, Tak PP. Synovial tissue response to rituximab: Mechanism of action and identification of biomarkers of response. Ann Rheum Dis 2008;67:917-25.

10. Arnett FC, Edworthy SM, Bloch DA, et al. The American Rheumatism Association 1987 revised criteria for the classification of rheumatoid arthritis. Arthritis Rheum 1988;312:315-24.

11. Prevoo ML, van't Hof MA, Kuper HH, Van Leeuwen MA, Van De Putte LB, Van Riel PL. Modified disease activity scores that include twenty-eight-joint counts: development and validation in a prospective longitudinal study of patients with rheumatoid arthritis. Arthritis Rheum 1995;38:44-8.

12. Rooney T, Bresnihan B, Andersson U, et al. Microscopic measurement of inflammation in synovial tissue: inter-observer agreement for manual quantitative, semiquantitative and computerised digital image analysis. Ann Rheum Dis 2007;66:1656-60

13. Haringman JJ, Vinkenoog M, Gerlag DM, Smeets TJ, Zwinderman AH, Tak PP. Reliability of computerized image analysis for the evaluation of serial synovial biopsies in randomized controlled trials in rheumatoid arthritis. Arthritis Res Ther 2005;7:R862-7.

14. Gerlag DM, Tak PP. Synovial biopsy. Best Pract Res Clin Rheumatol 2005;19:387-400.

15. Gerlag DM, Boyle DL, Rosengren S, Nash T, Tak PP, Firestein GS. Real-time quantitative PCR to detect changes in synovial gene expression in rheumatoid arthritis after corticosteroid treatment. Ann Rheum Dis 2007;66:545-7.

16. van der Pouw Kraan TC, Wijbrandts CA, van Baarsen LG, et al. Responsiveness to anti-TNF- $\alpha$ therapy is related to pre-treatment tissue inflammation levels in rheumatoid arthritis patients. Ann Rheum Dis 2008;67:563-6. 